\title{
A (In)sustentabilidade Hídrica Global e o Direito Humano à Água
}

\author{
Global Water (Un)sustainability and the Human Right to Water
}

\author{
Patricia Grazziotin Noschang ${ }^{1}$ \\ Adriana Fasolo Pilati Scheleder ${ }^{1}$ \\ ${ }^{1}$ Universidade de Passo Fundo, Passo Fundo - RS, Brasil.
}

Resumo: O uso inadequado dos recursos hídricos em algumas regiões do planeta, somado ao efeito trazido pelas mudanças climáticas, tem ocasionado períodos de seca e, consequentemente, a indisponibilidade hídrica levando à escassez. Considerando que a água é necessária para sobrevivência humana na Terra, os Estados devem garantir o acesso à água de qualidade para sua população. No entanto, essa garantia nem sempre é possível quando se percebe a má gestão dos recursos hídricos em alguns países. Essa é a questão discutida neste trabalho, que adota a abordagem dedutiva e a técnica bibliográfica e documental.

Palavras-chave: Crise Hídrica. Direito Humano à Água. Recursos Hídricos.
Abstract: The inadequate use of the water resources in some regions of the planet added to the effect brought by the climatic changes has caused periods of drought and consequently the water unavailability leading to the scarcity. Considering that water is necessary for human survival on Earth, States must guarantee access to quality water for their population. However, this guarantee is not always possible when the poor management of water resources is perceived in some countries. This is the issue faced by this work that adopts the deductive approach and the bibliographical and documentary technique.

Keywords: Water Crisis. Human Right to Water. Water Resources.

Recebido em: 04/01/2018

Revisado em: 04/07/2018

Aprovado em: 12/08/2018 


\section{Introdução}

A água é uma riqueza natural que garante a vida à humanidade na Terra. Historicamente, as comunidades se formaram perto ou nas nascentes dos rios, justamente para garantir a sua sobrevivência. Contudo, esse recurso natural é finito e por isso a utilização desse recurso deve ser de forma sustentável. Pode-se apontar como consequências da má utilização das águas, tanto superficiais como subterrâneas, o desaparecimento de um fluxo d'água (rio, lago, lagoa), o assoramento, a poluição, a eutrofização ${ }^{1}$, o desaparecimento dos seres vivos e micro-organismos, o consumo desordenado, entre outras.

À utilização inadequada dos recursos hídricos somam-se os efeitos causados pelas mudanças climáticas. Períodos de muita seca podem afetar a quantidade das águas e períodos de muita chuva podem atingir a qualidade das águas bem como provocar enchentes. Nesse sentido, todo recurso hídrico deve ser gerenciado adequadamente, por meio de políticas públicas estatais ou de atitudes da própria comunidade que o utiliza. Segundo Ribeiro (2008, p.19-20), a crise da água é resultado de uma série de fatores, entre eles: "[...] a escassez pontual, o consumo exagerado e elevação à condição de mercadoria em escala internacional”.

O objetivo de uma gestão adequada e integrada de recursos hídricos é, pois, garantir a disponibilidade da água e, em decorrência, a vida da humanidade. Muitas regiões do mundo já estão enfrentando problemas com a escassez de água em consequência do mau uso dos recursos hídricos e dos

\footnotetext{
${ }^{1}$ Segundo José Galizia Tundisi (2005, p. 67), “A eutrofização dos ecossistemas aquáticos continentais, das águas costeiras marinhas e das águas subterrâneas é resultado do enriquecimento com nutrientes de plantas, principalmente o fósforo e nitrogênio, que são despejados de forma dissolvida ou particulada em lagos, represas e rios e são transformados em partículas orgânicas, matéria viva vegetal, pelos metabolismos das plantas. A eutrofização natural é resultado da descarga normal de nitrogênio e fósforo nos sistemas aquáticos. A eutrofização 'cultural' é proveniente dos despejos de esgotos domésticos e industriais e da descarga de fertilizantes aplicados na agricultura [...]. No caso de lagos, represas e rios, esse processo consiste no rápido desenvolvimento de plantas aquáticas, inicialmente cianobactérias, ou 'algas verdes azuis', as quais produzem substâncias tóxicas que podem afetar a saúde do homem e podem causar a mortalidade de animais e intoxicações".
} 
efeitos das mudanças climáticas. A gestão e o consumo inadequado da água poderá levar a indisponibilidade hídrica e consequentemente a escassez.

A escassez hídrica tem provocado o que se conhece hoje como crise da água, estresse hídrico ou crise hídrica. A crise hídrica já uma questão séria, posta e suas consequências podem se tornar ainda mais sérias. Dos Estados Unidos da América ao Brasil (com destaque para o Estado de São Paulo, que, desde 2014, amarga uma das maiores crises hídricas), da África ao Oriente Médio e também na Europa, os países enfrentam o problema da falta de água para abastecimento da sua população em diversas regiões.

Nesse sentido, enquanto os Estados devem garantir a água a sua população por ser um direito humano, reconhecido internacionalmente, também tem como obrigação a gestão adequada desse recurso natural para que a água seja suficiente e disponível a todos de forma igualitária para consumo. Este trabalho busca apresentar como a água se tornou um direito humano bem como demonstrar que esse recurso natural é finito e, depende de um consumo sustentável e uma gestão adequada para estar disponível às futuras gerações.

\section{O Direito Humano à Água}

O entendimento de que a água é indispensável para a sobrevivência do ser humano neste planeta permite vislumbrar a existência de um direito humano fundamental ao acesso à água potável. A água é um recurso natural que deve estar disponível e ser compartilhado por toda a população mundial. Para assegurar esse direito fundamental, caberá aos Estados gerenciar o acesso e a disponibilidade da água nos limites da sua soberania.

É recente o reconhecimento da água como um direito humano. Os principais documentos relacionados à proteção dos direitos humanos ainda não a incluíram no rol de seus princípios ou bens que necessitam ser protegidos. Essa lacuna é notada na Declaração Universal de Direitos Humanos de 1948, que não menciona a questão da água, nem mesmo os Pactos Internacionais de Direitos Civis e Políticos e o de Direitos Econômicos Sociais e Culturais, ambos elaborados em 1966. 
O Pacto Internacional de Direitos Civis e Políticos e o Pacto Internacional de Direitos Econômicos, Sociais e Culturais, disponibilizados para assinatura (adesão) em 1966, foram elaborados com o objetivo de dar efetividade à Declaração Universal de Direitos Humanos “[...] uma vez que a DUDH ficou no plano dos princípios, sem mecanismos de cobrança aos Estados" (NOSCHANG, 2013, p. 256-257).

Para cuidar da aplicação dos preceitos previstos em ambos os Pactos, foram criados órgãos incumbidos desta função. No caso dos direitos civis e políticos, a responsabilidade recaiu sobre o Comitê de Direitos Humanos $^{2}$; os direitos econômicos, sociais e culturais ficaram a cargo do comitê respectivo (Comitê de Direitos Econômicos, Sociais e Culturais) ${ }^{3}$. 2 "The Human Rights Committee is the body of independent experts that monitors
implementation of the International Covenant on Civil and Political Rights by its State
parties. All States parties are obliged to submit regular reports to the Committee on how
the rights are being implemented. States must report initially one year after acceding
to the Covenant and then whenever the Committee requests (usually every four years).
The Committee examines each report and addresses its concerns and recommendations
to the State party in the form of 'concluding observations'. In addition to the reporting
procedure, article 41 of the Covenant provides for the Committee to consider inter-state
complaints. Furthermore, the First Optional Protocol to the Covenant gives the Committee
competence to examine individual complaints with regard to alleged violations of the
Covenant by States parties to the Protocol. The full competence of the Committee extends
to the Second Optional Protocol to the Covenant on the abolition of the death penalty with
regard to States who have accepted the Protocol. The Committee meets in Geneva and
normally holds three sessions per year. The Committee also publishes its interpretation
of the content of human rights provisions, known as general comments on thematic
issues or its methods of work". (UNITED NATIONS HUMAN RIGHTS. Human Rights
Committee, 2017a)

3 "The Committee on Economic, Social and Cultural Rights (CESCR) is the body of independent experts that monitors implementation of the International Covenant on Economic, Social and Cultural Rights by its States parties. The Committee was established under ECOSOC Resolution 1985/17 of 28 May 1985 to carry out the monitoring functions assigned to the United Nations Economic and Social Council (ECOSOC) in Part IV of the Covenant. All States parties are obliged to submit regular reports to the Committee on how the rights are being implemented. States must report initially within two years of accepting the Covenant and thereafter every five years. The Committee examines each report and addresses its concerns and recommendations to the State party in the form of "concluding observations". In addition to the reporting procedure, the Optional Protocol to the International Covenant on Economic, Social and Cultural Rights, which 
A inclusão da água como garantia de um direito para a sobrevivência humana ocorreu somente em 2002, com o General Comments $n$. 15 do Comitê de Direitos Econômicos Sociais e Culturais. Quase dez anos depois, em 2010, a Assembleia Geral da ONU adotou uma Resolução, considerada histórica, reconhecendo o direito humano à água limpa e potável e ao saneamento, como essencial para o pleno gozo do direito à vida.

O Comitê de Direitos Sociais Econômicos e Culturais foi o responsável por incluir a água como um direito à sobrevivência humana como assentado no Comentário Geral n. 15 (General Comments n.15 - GC15) de interpretação aos artigos $11^{4}$ e $12^{5}$ do Pacto de Direitos Econômicos

entered into force on 5th May 2013, provides the Committee competence to receive and consider communications from individuals claiming that their rights under the Covenant have been violated. The Committee may also, under certain circumstances, undertake inquiries on grave or systematic violations of any of the economic, social and cultural rights set forth in the Covenant, and consider inter-state complaints. The Committee meets in Geneva and normally holds two sessions per year, consisting of a three-week plenary and a one-week pre-sessional working group. The Committee also publishes its interpretation of the provisions of the Covenant, known as general comments. (UNITED NATIONS HUMAN RIGHTS. Committee on Economic, Social and Human Rights, 2017a)

4 "Artigo 11. 1. Os Estados Partes do presente Pacto reconhecem o direito de toda pessoa a um nível de vida adequando para si próprio e sua família, inclusive a alimentação, vestimenta e moradia adequadas, assim como a uma melhoria contínua de suas condições de vida. Os Estados Partes tomarão medidas apropriadas para assegurar a consecução desse direito, reconhecendo, nesse sentido, a importância essencial da cooperação internacional fundada no livre consentimento. 2. Os Estados Partes do presente Pacto, reconhecendo o direito fundamental de toda pessoa de estar protegida contra a fome, adotarão, individualmente e mediante cooperação internacional, as medidas, inclusive programas concretos, que se façam necessárias para: a) Melhorar os métodos de produção, conservação e distribuição de gêneros alimentícios pela plena utilização dos conhecimentos técnicos e científicos, pela difusão de princípios de educação nutricional e pelo aperfeiçoamento ou reforma dos regimes agrários, de maneira que se assegurem a exploração e a utilização mais eficazes dos recursos naturais; b) Assegurar uma repartição eqüitativa dos recursos alimentícios mundiais em relação às necessidades, levando-se em conta os problemas tanto dos países importadores quanto dos exportadores de gêneros alimentícios." (BRASIL. Decreto n. 591, de 6 de julho de 1992, 2017)

5 "ARTIGO 12. 1. Os Estados Partes do presente Pacto reconhecem o direito de toda pessoa de desfrutar o mais elevado nível possível de saúde física e mental. 2. As medidas que os Estados Partes do presente Pacto deverão adotar com o fim de assegurar o pleno 
Sociais e Culturais, em 2002 (RIBEIRO, 2008, p. 111). O documento determina que a água é pré-requisito para a realização dos outros direitos humanos, pois o direito humano à água é indispensável para uma vida com dignidade. Ademais, vaticina que a água é um recurso natural limitado e um bem público fundamental para vida e a saúde.

A propósito, no entendimento do GC-15,

[...] o direito à água consiste no abastecimento suficiente, fisicamente acessível e a um custo compatível, de uma água salubre de qualidade aceitável para os usos pessoais e domésticos de cada um. (UNITED NATIONS ECONOMIC AND SOCIAL COUNCIL, 2012, p. 2-3)

O direito à água já foi reconhecido por uma série de documentos internacionais, incluindo tratados, resoluções e outras decisões nesse âmbito $^{6}$. Entre esses documentos estão a "Convenção sobre Eliminação de

exercício desse direito incluirão as medidas que se façam necessárias para assegurar: a) A diminuição da mortinatalidade e da mortalidade infantil, bem como o desenvolvimento é das crianças; b) A melhoria de todos os aspectos de higiene do trabalho e do meio ambiente; c) A prevenção e o tratamento das doenças epidêmicas, endêmicas, profissionais e outras, bem como a luta contra essas doenças; d) A criação de condições que assegurem a todos assistência médica e serviços médicos em caso de enfermidade." (BRASIL. Decreto n. 591, de 6 de julho de 1992).

${ }^{6}$ Arts. 20, 26, 29 and 46 of the Geneva Convention relative to the Treatment of Prisoners of War, of 1949; arts. 85, 89 and 127 of the Geneva Convention relative to the Treatment of Civilian Persons in Time of War, of 1949; arts. 54 and 55 of Additional Protocol I thereto of 1977; arts. 5 and 14 Additional Protocol II of 1977; preamble, Mar Del Plata Action Plan of the United Nations Water Conference; see para. 18.47 of Agenda 21, Report of the United Nations Conference on Environment and Development, Rio de Janeiro, 3-14 June 1992 (A/CONF.151/26/Rev.1 (Vol. I and Vol. I/Corr.1, Vol. II, Vol. III and Vol. III/Corr.1) (United Nations publication, Sales No. E.93.I.8), vol I: Resolutions adopted by the Conference, resolution 1, annex II; Principle No. 3, The Dublin Statement on Water and Sustainable Development, International Conference on Water and the Environment (A/ CONF.151/PC/112); Principle No. 2, Programme of Action, Report of the United Nations International Conference on Population and Development, Cairo, 5-13 September 1994 (United Nations publication, Sales No. E.95.XIII.18), chap. I, resolution 1, annex; paras. 5 and 19, Recommendation (2001) 14 of the Committee of Ministers to Member States on the European Charter on Water Resources; resolution 2002/6 of the United Nations Sub- 
todas as formas de Discriminação contra as Mulheres" e a "Convenção sobre os Direitos das Crianças". Na primeira, o direito à água é considerado em termos de acesso, uma vez que muitas mulheres, em algumas regiões do mundo, precisam percorrer longas distâncias para encontrar fontes de água potável para a sua sobrevivência e a de sua família. Na segunda, a relação entre a água e as crianças está ancorada na prevenção de doenças e na má nutrição ${ }^{7}$ (UNITED NATIONS. ECONOMIC AND SOCIAL COUNCIL, 2012, p. 2-3).

Ainda, segundo o GC-15, a prioridade da disponibilidade da água deve ser dada ao uso pessoal e doméstico bem como para prevenir a fome e as doenças (UNITED NATIONS. ECONOMIC AND SOCIAL COUNCIL, 2012, p. 3).

Ressalta-se que a água é considerada e deve ser tratada como um bem social e cultural e não como um bem econômico, isso para garantir que a realização do direito à água seja sustentável e que esteja disponível para as presentes e as futuras gerações (UNITED NATIONS. ECONOMIC AND SOCIAL COUNCIL, 2012, p. 5). A água, além disso, não deve ser usada como um instrumento de pressão política e econômi$\mathrm{ca}^{8}$ (UNITED NATIONS. ECONOMIC AND SOCIAL COUNCIL, 2012, p. 11-12). A determinação do GC-15 aponta nesse sentido quando deter-

Commission on the Promotion and Protection of Human Rights on the promotion of the realization of the right to drinking water. See also the report on the relationship between the enjoyment of economic, social and cultural rights and the promotion of the realization of the right to drinking water supply and sanitation (E/CN.4/Sub.2/2002/10) submitted by the Special Rapporteur of the Sub-Commission on the right to drinking water supply and sanitation, Mr. El Hadji Guissé. (UNITED NATIONS ECONOMIC AND SOCIAL COUNCIL. General Comment 15. E/C.12/2002/11. Geneva, 2012, p. 2-3.)

${ }^{7}$ Art. 14, par. $2(h)$, Convention on the Elimination of All Forms of Discrimination Against Women; art. 24, par. 2 (c), Convention on the Rights of the Child. (UNITED NATIONS. Economic and Social Council, 2012, p. 2-3)

${ }^{8}$ A este respeito, a Comissão recorda a sua posição sobre a relação entre as sanções econômicas e de respeito aos direitos econômicos sociais e culturais: "In General Comment No. 8 (1997), the Committee noted the disruptive effect of sanctions upon sanitation supplies and clean drinking water, and that sanctions regimes should provide for repairs to infrastructure essential to provide clean water.". (UNITED NATIONS. ECONOMIC AND SOCIAL COUNCIL. General Comment 15. E/C.12/2002/11. Geneva, 2012, p. 11-12.) 
mina que: "Os Estados Partes devem adotar medidas efetivas para concretizar, sem discriminação, o direito à água, conforme estabelecido neste comentário geral" (UNITED NATIONS. ECONOMIC AND SOCIAL COUNCIL, 2012, p. 2).

A importância do GC15 reside justamente na iniciativa de incluir a água como um direito humano e bem essencial ao direito à vida. Esse foi o primeiro passo contra pressões políticas e econômicas que buscam o domínio da água, considerando-a como bem econômico e, consequentemente, pretendem a sua apropriação privada.

Nessa esteira, também quadra destacar que a Assembleia Geral da ONU, quase dez anos após a emissão do GC15, venceu outra batalha contra o lobby econômico da água ao aprovar a Resolução n. 64/292 sobre o direito humano à água e ao saneamento.

A contribuição da Assembleia Geral da ONU veio em 2010, com a aprovação de uma resolução no plenário, em uma sessão tensa motivada pela oposição de um número expressivo de Estados "poderosos"10 (BARLOW, 2012, p. XV). Pela Resolução n. 64/292, reconheceu-se o direito à água e ao saneamento "para o pleno gozo do direito à vida" (BARLOW, 2012, p. XV) Dois meses depois, o Conselho de Direitos Humanos da ONU também aprovou a Resolução A/HRC/15/L.14, na qual afirmava que água e o saneamento são direitos humanos que estão atrelados ao direito a um padrão adequado de vida, com saúde física e mental (UNITED NATIONS. GENERAL ASSEMBLY, 2017b).

9 "States parties have to adopt effective measures to realize, without discrimination, the right to water, as set out in this general comment." (UNITED NATIONS. ECONOMIC AND SOCIAL COUNCIL. General Comment 15. E/C.12/2002/11. Geneva, 2012, p. 2.) 10 "Bolivian UN Ambassador Pablo Solon intoduced the resolution by reminding the assembly that humans are about two-thirds made of water and our blood flows like a network of rivers to transport nutrients and energy to our bodies. 'Water is life', he said. But then he laid out the tragic and growing numbers of people around the world dying from lack of access to clean water and quoted a new World Health Organization study on diarrhoea showing that every three and a half seconds in the developing world, a child dies of water-borne disease. Ambassador Solon then quietly snapped his fingers three times and held his small finger up for half second. The General Assembly of United Nations fell silent. Moments later, it voted overwhwlmingly to recognize the human right to water and sanitation. The floor erupted in cheers." (BARLOW, 2012. p. XV.) 
Barlow (2012), sobre o tema, conta que ambas as resoluções representam uma grande conquista na luta pelo direito à água potável e ao saneamento, configurando um marco crucial na busca da justiça pela água. Acrescenta que elas também complementam a promessa realizada na Conferência da Terra em 1992, na cidade do Rio de Janeiro, na qual a água, as mudanças climáticas, a biodiversidade e a desertificação foram alvos de ações. A luta por esse marco fundamental é antiga e foi bloqueada diversas vezes por corporações poderosas e governos, que preferiam ver a água como uma mercadoria privada para ser colocada à venda no mercado, tanto que 41 Estados-membros se abstiveram de votar a resolução na Assembleia Geral da ONU (BARLOW, 2012, p. XV-XVI).

A justiça pela água, referida por Barlow, está vinculada à disponibilidade de água potável, ao acesso à água e ao saneamento por todos os seres humanos e à garantia de que esse recurso natural estará disponível para as atuais e as futuras gerações (equidade intergeracional). Cada Estado deve, assim, garantir que sua população tenha acesso à água e ao saneamento. No entanto, com a crise hídrica que assola alguns Estados ou regiões esse dever fica prejudicado. É nesse sentido que se intenta considerar a água uma preocupação comum da humanidade e construir a concepção do dever de cooperação e de solidariedade. Com esta proposta, objetiva-se a preservação dos recursos hídricos para garantir a sobrevivência das futuras gerações. Essa proposta depende também da contraprestação do indivíduo como consumidor final da água. Faz-se necessário também que a população contribua com o consumo sustentável da água com condutas e formando uma consciência global para evitar o desperdício na sua utilização.

\section{Disponibilidade Hídrica e Consumo Global}

Os conceitos de "escassez hídrica" ou "estresse hídrico" passaram a ser debatidos e foram relacionados diretamente com o consumo mínimo das águas que se considera necessário para cada ser humano na Terra prover suas necessidades com qualidade. De acordo com Ribeiro (2008, p. 55), “[...] quantificar o volume mínimo necessário a uma vida digna passou a ser uma obstinação de muitos autores e organismos internacionais". 
A escassez hídrica “[...] é uma das medidas de avaliação geográfica de uma unidade territorial. Ela pode ser física e econômica" (RIBEIRO, 2008 , p. 62). Na verdade, não há um conceito único de crise hídrica pois tal definição depende dos elementos a serem considerados pelos estudiosos para formá-lo, somado o fato de que cada autor considera diferentes critérios que entende relevantes para definir crise hídrica, entre eles: Malin Falkeenmark (1989) - para quem o índice de estresse hídrico resulta da divisão da água disponível, por país, pelo total da população; Ohlsson (1999) - que desenvolveu o Índice Social de Estresse Hídrico; Peter Gleick (1993) (PACIFIC INSTITUTE, 2017) - mencionou o estresse hídrico e definiu as regiões de risco de conflitos internacionais por água (RIBEIRO, 2008, p. 63).

Em se tratando de medir o estresse hídrico, logicamente, cada classificação apresenta vantagens e desvantagens. É como explica Ribeiro (2008, p. 71):

Cada classificação proposta para quantificar a escassez ou o estresse hídrico oferece vantagens e dificuldades. O conceito de escassez hídrica aponta lugares onde existe dificuldade de acesso à água em quantidade e qualidade adequada. Já o estresse hídrico depende da informação correta do volume consumido. Isso exige um rigoroso sistema para quantificar o volume de água usado todos os anos, o que também não é simples nem barato. $O$ fator de uso do fluxo da bacia é o indicador mais complexo porque exige um apurado conhecimento da dinâmica de cada bacia hidrográfica, além de não distinguir os usos da água. Se $70 \%$ da água de uma bacia for usada para gerar hidroeletricidade, por exemplo, seu reuso pode ser total se forem tomados os devidos cuidados após sua passagem pelas turbinas.

Segundo Shiva (2006, p. 17),

[...] diz-se que um país enfrenta uma crise de água quando a água disponível é menos que mil metros cúbicos por habitante ano. Abaixo desse ponto, a saúde e o desenvolvimento econômico de uma nação são dificultados consideravelmente. 
Afirma ainda que quando a disponibilidade de água anual por habitante cai abaixo de quinhentos metros cúbicos, a sobrevivência da população é cruelmente comprometida (SHIVA, 2006, p. 17).

Já para Ribeiro (2008, p. 71), o estresse hídrico é a pressão exercida pela falta de água, enquanto a escassez representa a efetiva falta de água. Dentro de uma escala progressiva, o estresse vem primeiro que a escassez, mas ambas as situações causam sérios problemas (RIBEIRO, 2008).

Os fatores que influenciam a escassez de água disponível para a população são o aumento da população, a irrigação utilizada na agricultura e o crescimento econômico desordenado. Esse conjunto de ações produzidas pelas atividades humana, no afã de explorar os recursos hídricos para expandir o desenvolvimento econômico e fazer frente às demandas industriais e agrícolas e à expansão e ao crescimento da população e das áreas urbanas acabou tornando-se um problema complexo ao longo da história (TUNDISI, 2005, p. 35).

O Gráfico 1, a seguir, demonstra os índices de retirada de água nos setores que mais utilizam a água como suprimento.

Gráfico 1: Índices de retirada de água, por continente

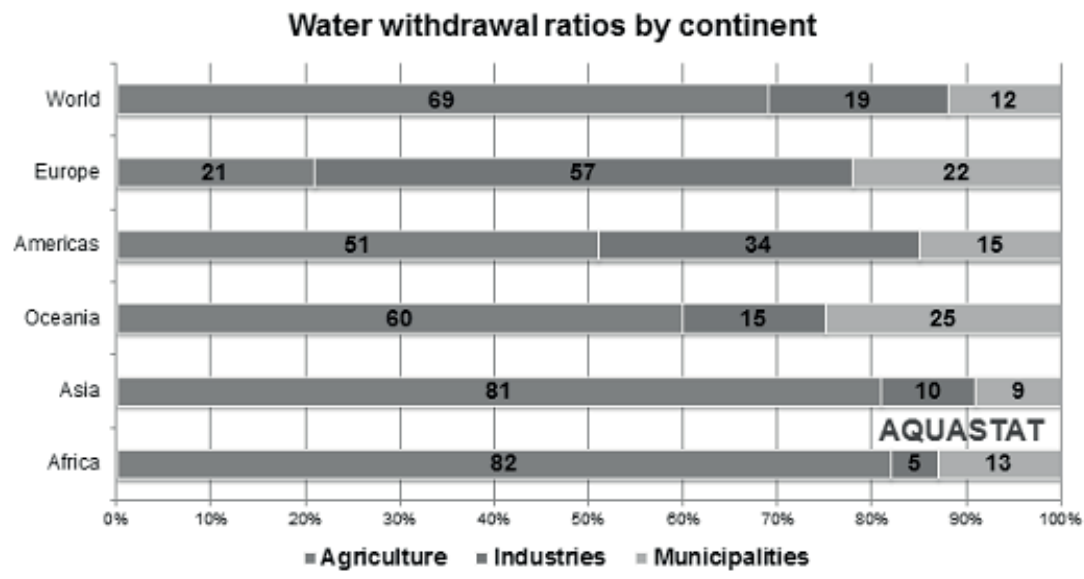

Fonte: Food Agriculture Organization of United Nationas - Aquastat (2017) 
O Gráfico 2 apresenta o crescimento populacional e, consequentemente, o aumento da demanda hídrica ao longo dos anos.

Gráfico 2: População mundial e retirada de água ao longo do tempo

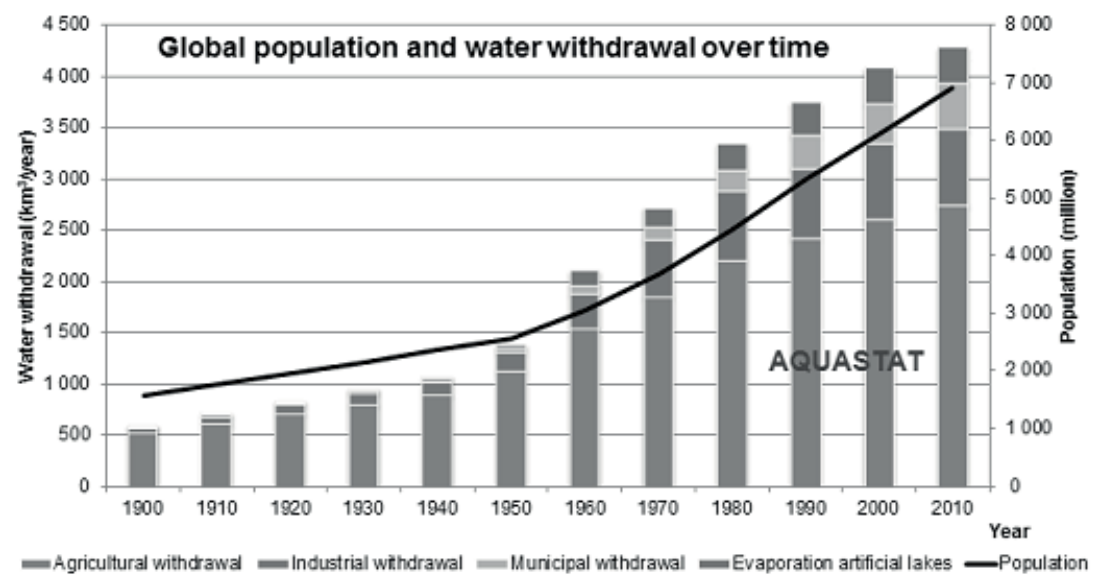

Fonte: Food Agriculture Organization of United Nationas - Aquastat (2017)

Nos gráficos apresentados percebe-se que a agricultura é o setor campeão de uso de água. A água utilizada na produção agrícola, comercializada em termos globais, é considerada como uma exportação "virtual". A pecuária é outro setor que também utiliza grande quantidade de água para produção e exportação da carne. Nesse sentido, é possível afirmar que existe um consumo virtual de água quando esses produtos chegam aos países importadores e são disponibilizados ao consumidor final.

Segundo Hoekstra e Chapagain (2008, p. 8-12), água virtual é todo volume de água doce utilizado para produzir um produto ao longo das várias etapas da cadeia de produção. O adjetivo "virtual" se refere à água utilizada para a produção, mas que não está contida no produto em si. Segundo os autores, o teor de água real dos produtos é praticamente insignificante se comparado como o teor de água virtual utilizada na produção (HOEKSTRA; CHAPAGAIN, 2008). Apenas para ilustrar, são necessários 13 litros de água para produzir um único tomate; 1.000 litros de água são necessários para produzir um quilo de pão; e mais de 14.000 litros são necessários para produzir um quilograma de um bife de gado (WINK- 
LER, 2012, p. 28). Os referidos autores também criaram o conceito de pegada hídrica, que representa o volume total anual de água utilizado na produção de um bem ou serviço consumido por um grupo de consumidores incluindo uma família, empresa, cidade, Estado ou nação.

A Figura 1 demonstra a quantidade de litros de água necessários para produzir alguns produtos.

Figura 1: Número de litros de água necessário para a produção de alimentos
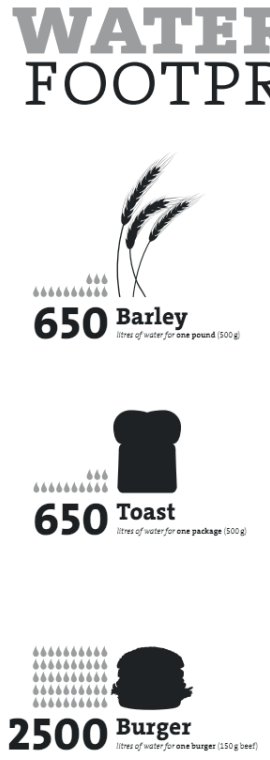

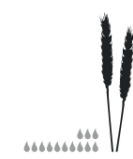

650 wheat
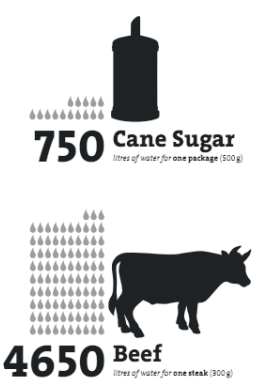
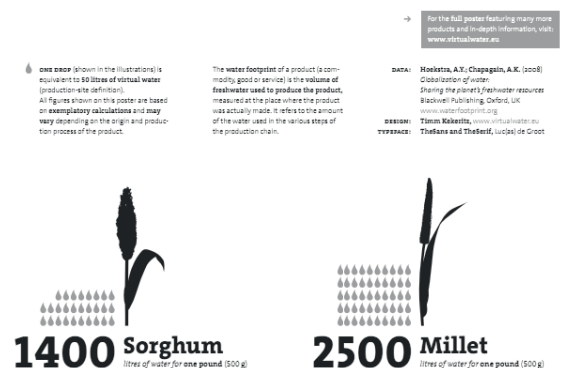

1400 Sorghum

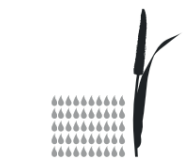

2500 Millet
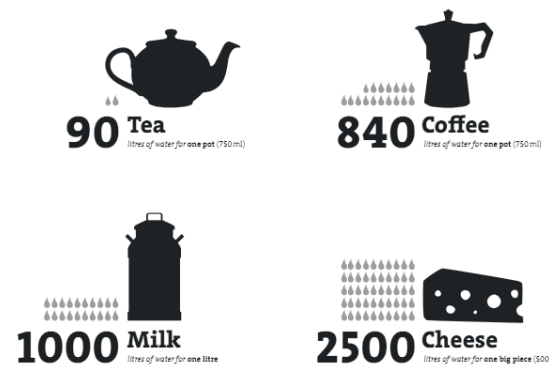

Fonte: Virtual Water (2017)

Alguns países decidiram importar alimentos que utilizam uma grande quantidade de água para produção devido à indisponibilidade hídrica no seu território. A escassez hídrica determinou que países como o Egito e a Jordânia, por exemplo, importassem os principais alimentos para suprir a sua população. Esses países, portanto, passam a ser importadores de água virtual. Por outro lado, países com disponibilidade hídrica são os grandes exportadores de água virtual; é o caso de Índia, Argentina, Estados Unidos, Austrália e Brasil (FREITAS, 2017). 
O Brasil é considerado o quinto maior exportador de água virtual do mundo (FREITAS, 2017). O relatório da Agência Nacional de Águas (ANA), denominado Relatório de Conjuntura dos Recursos Hídricos, que apresentou dados comparativos ao uso da água no Brasil no período de 2006 a 2010, permite que se entenda que em quase todas as bacias hidrográficas brasileiras houve um aumento da retirada de água, principalmente para a irrigação (BRASIL, ANA, 2013).

Gráfico 3: Distribuição da demanda de água (usos nas regiões hidrográficas do Brasil)

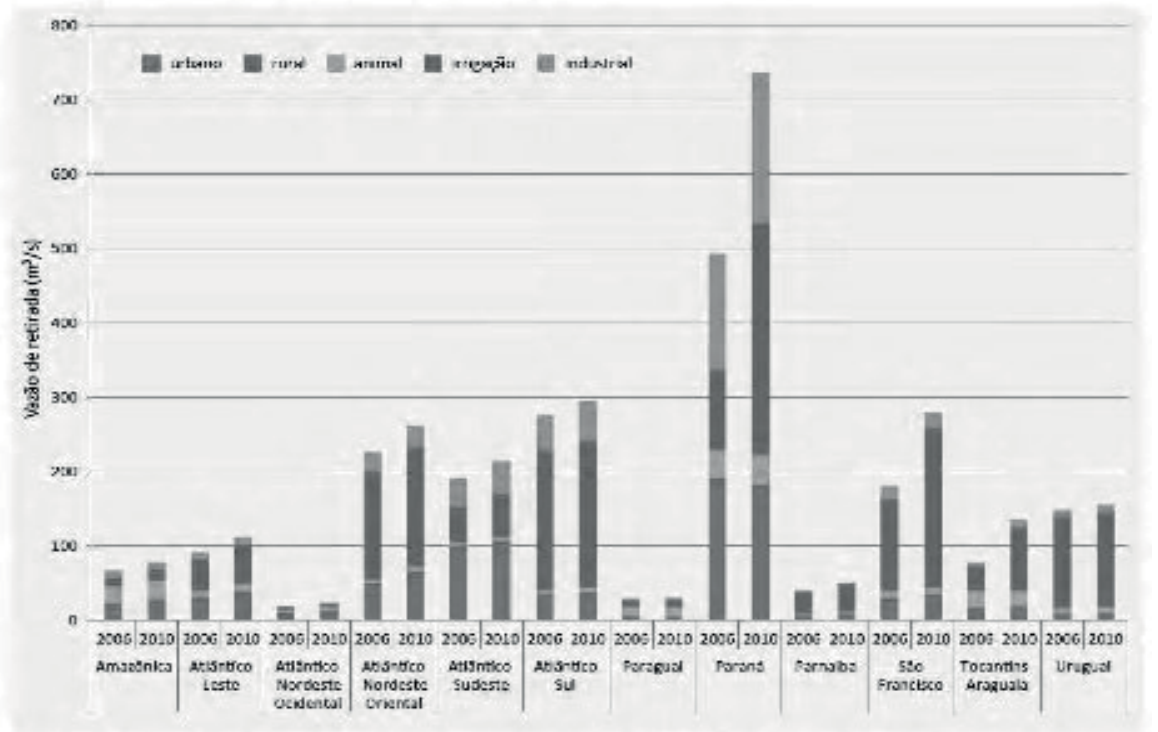

Fonte: Brasil - ANA (2013)

Bom exemplo da falta de planejamento do governo na utilização dos recursos hídricos foi a redução do Mar de Aral. Localizado na Ásia Central, na República do Cazaquistão, em 1960, esse lago possuía uma profundidade média de 53,4 metros, área de $66.900 \mathrm{~km}^{2}$ e volume de $1.050 \mathrm{~km}^{3}$. O projeto de estabelecer e expandir a produção de algodão irrigado pelas águas do Mar de Aral trouxe a dependência da monocultura e da irrigação aos países da Ásia Central, onde a demanda aumentou consideravelmente em trinta anos. Em consequência, a área do Mar de Aral reduziu para $31.938 \mathrm{~km}^{2}$, em 1994, e para $25.217 \mathrm{~km}^{2}$, em 2000. A redução drástica do volume de água fez com que a salinidade aumen- 
tasse de $10 \mathrm{~g} / \mathrm{L}$, em 1960, para 60 g/L, em 2000. Não bastasse, o aumento da salinidade e a utilização excessiva de pesticidas tornaram o solo tóxico e inutilizado. À catástrofe natural somaram-se as consequências econômicas e sociais desse ambicioso projeto governamental:

[...] a morbidez aumentou $29 \%$ em razão de doenças resultantes de substâncias tóxicas e da salinização. Pesticidas, fertilizantes e outras substâncias carcinogênicas aumentaram em 50\% o índice de câncer. (TUNDISI, 2005, p. 46-47)

A indústria da pesca desapareceu e deixou milhares de desempregados, e cerca de 35 milhões de pessoas foram afetadas pelo desastre (TUNDISI, 2005). A população da região teve que buscar novas alternativas de consumo e sobrevivência pois quem dependia das águas do Mar de Aral foi obrigado a migrar para outros locais.

O Relatório da Organização das Nações Unidas para a Educação, a Ciência e a Cultura (United Nations Educational Scientific and Cultural Organization - UNESCO), de 2015, também reconhece que as consequências de um desenvolvimento/crescimento insustentável e as falhas de políticas governamentais que atingem diretamente a disponibilidade dos recursos hídricos comprometem sobremaneira a geração de benefícios sociais e econômicos, bem como o consumo da água para sobrevivência humana. A demanda pela água doce continua aumentando e se o equilíbrio entre demanda e oferta não for restaurado o mundo enfrentará um déficit global de água cada vez mais grave. O relatório reforça a influência do crescimento populacional, da urbanização, das políticas de segurança alimentar e energética, e dos processos macroeconômicos, como globalização do comércio, mudanças na dieta e aumento do consumo na demanda hídrica global. Também prevê para 2050 um aumento da demanda hídrica mundial da ordem de $55 \%$, principalmente devido à crescente demanda oriunda do setor industrial, dos sistemas de geração de energia termoelétrica e dos usuários domésticos (UNITED NATIONS EDUCATIONAL, SCIENTIFIC AND CULTURAL ORGANIZATION, 2017, p. 3 ).

Em alguns países, a retirada de água tem aumentado significativamente, causando um desequilíbrio no ciclo hidrológico. Isso ocorre tanto 
em águas superficiais como nas subterrâneas, quando o uso da água para irrigação, para fins industriais ou mesmo abastecimento público vai além da quantidade de reposição por precipitação e recarga. A consequência é, com toda evidência, desequilíbrio e escassez.

Os exemplos são inúmeros, valendo citar: no Norte da China, onde retiradas de águas subterrâneas excederam a recarga do aquífero, o lençol freático, em algumas áreas, sofreu reduções de 1 a 4 metros por ano. Em High Plains, Estados Unidos, “[...] o aquífero de Ogallala, que supre água de irrigação para essas regiões, está diminuindo e, em algumas áreas, já perdeu 50\% do volume de água" (TUNDISI, 2005, p.54-55). Na Califórnia, as águas do Owens Valley e da bacia hidrográfica do Mono Lake foram utilizadas para o suprimento de usuários no sul do estado. Resultado: o primeiro secou e o segundo diminuiu um terço (TUNDISI, 2005).

Os dados disponíveis sobre o consumo anual de água no mundo e da quantidade de água renovável, conforme avalia Ribeiro (2008), demonstram que não há falta de água quando se levam em consideração os processos naturais que geram a oferta hídrica. Entretanto, quando a análise é feita por país, essa indisponibilidade é identificada.

Além disso, mesmo sem água no território é possível conseguir esta substância por meios econômicos ou políticos, como a guerra. Isso permite afirmar que a falta de água não é um problema natural, mas político. (RIBEIRO, 2008, p. 72)

Soma-se ao problema político apontado por Ribeiro, o crescimento não planejado das cidades, as mudanças climáticas e a falta de consciência da população no consumo sustentável da água em cada residência.

\section{Conclusão}

A luta pelo direito humano à água formou um movimento denominado justiça pela água que está vinculado ao acesso à água, ao saneamento e à disponibilidade de água potável para todos os seres humanos, bem como para as atuais e futuras gerações. O direito humano à água foi reco- 
nhecido por vários documentos internacionais, porém, sob árdua labuta. Assim, é dever de cada Estado garantir a sua população o acesso à água e ao saneamento.

Se, por um lado, o Estado deve garantir a sua população o acesso à água e ao saneamento, por outro, o indivíduo tem como obrigação o consumo sustentável desse recurso natural finito. É necessário que o ser humano tome consciência que não há água em abundância no planeta e a ele cabe a responsabilidade de usar esse recurso natural adequadamente evitando o desperdício.

Os Estados, em âmbito interno, devem realizar a gestão sustentável de seus recursos naturais considerando que a água é um recurso finito e que existem influências climáticas que devem ser observadas para evitar a escassez hídrica e consequentemente uma crise hídrica. Nesse sentido, as mudanças climáticas podem interferir na disponibilidade hídrica dos Estados, tanto para períodos de abundância quanto para períodos de escassez.

Todavia, quando as águas (rios, lagos ou aquíferos) são transfronteiriços a gestão hídrica sustentável desses recursos hídricos deverá ser compartilhada entre os Estados ribeirinhos aplicando sempre o princípio da cooperação e da precaução para que a gestão seja eficiente e o meio ambiente considerado.

\section{Referências}

BARLOW, Maude. Foreword. In: SULTANA, Farhana; LOFTUS, Alex. (Org.) The right to water: politics, governance and social struggles, Oxon: Earthscan, 2012. p. XI-XVIII.

BRASIL. Decreto n. 591, de 6 de julho de 1992. Atos Internacionais. Pacto Internacional sobre Direitos Econômicos, Sociais e Culturais. Promulgação. Disponível em: < http://www.planalto.gov.br/ccivil_03/ decreto/1990-1994/D0591.htm>. Acesso em: 10 abr. 2017.

BRASIL - ANA. Agência Nacional de Águas. Conjuntura dos recursos hídricos no Brasil. Brasília: ANA, 2013. 
FOOD AGRICULTURE ORGANIZATION OF UNITED NATIONS. AQUASTAT. Water uses. [2017]. Disponível em: <http://www.fao.org/ nr/water/aquastat/water_use/index.stm>. Acesso em: 25 set. 2017.

FREITAS, Tatiana. Brasil é o $5^{\circ}$ maior exportador de "água virtual" incorporada a alimento. Folha de São Paulo. 20 de março de 2015. Disponível em: <http://www1.folha.uol.com.br/ mercado/2015/03/1605650-brasil-e-o-5-maior-exportador-de-aguavirtual-incorporada-a-alimentos.shtml>. Acesso em: 25 set. 2017.

Hoekstra, Arjen; Chapagain, Ashok. Globalization of water: sharing the planet's freshwater resources. Oxford: Wiley-Blackwell, 2008. p. 8-12.

NOSCHANG, Patricia Grazziotin. Os sistemas de proteção aos direitos humanos e o Brasil: da participação no sistema global ao (des)cumprimento na esfera regional. Revista Direitos Humanos e Democracia, Ijuí: Unijuí, V. 1, p. 250-280, 2013.

PACIFIC INSTITUTE. Water conflict chronology. 2009. Disponível em: <www.pacinst.org>. Acesso em: 28 set. 2017.

RIBEIRO, Wagner C. Geografia política da água. São Paulo: Annablume, 2008.

SHIVA, Vandana. Guerra por água: privatização, poluição e lucro. São Paulo: Radical Livros, 2006.

TUNDISI, José Galizia. Água no Seculo XXI: enfrentando a escassez. 2. ed. São Carlos: RiMa, 2005.

UNITED NATIONS HUMAN RIGHTS. Human Rights Committee. Introdution. [2017a]. Disponível em: <http://www.ohchr.org/EN/ HRBodies/CCPR/Pages/CCPRIntro.aspx>. Acesso em: 10 abr. 2017.

UNITED NATIONS HUMAN RIGHTS. Committee on Economic, Social and Human Rights. [2017b]. Disponível em: $<$ http://www.ohchr. org/EN/HRBodies/CESCR/Pages/CESCRIntro.aspx>. Acesso em: 10 abr. 2017. 
UNITED NATIONS. General Assembly. A/RES/64/292. The human right to water and sanitation. [2017b]. Disponível em: $<$ http://www. un.org/en/ga/search/view_doc.asp?symbol=A/RES/64/292>. Acesso em: 28 set. 2017.

UNITED NATIONS. General Assembly. A/HRC/15/L.14. Human Rights Council. Human rights and access to safe drinking water and sanitation. [2017b]. Disponível em: $<$ http://www.internationalwaterlaw. org/documents/intldocs/UNGA-HRC_Resolution-HR_to_Water_and_ Sanitation.pdf $>$. Acesso em: 28 set. 2017.

UNITED NATIONS. Economic and Social Council. General Comment 15. E/C.12/2002/11. Geneva, 2012.

UNITED NATIONS EDUCATIONAL, SCIENTIFIC AND CULTURAL ORGANIZATION. Relatório Mundial das Nações Unidas sobre Desenvolvimento dos Recursos Hídricos. Água para um mundo sustentável. Sumário Executivo, p. 3. 2015. Disponível em: $<$ http:// www.unesco.org/new/fileadmin/MULTIMEDIA/HQ/SC/images/ WWDR2015ExecutiveSummary_POR_web.pdf $>$. Acesso em: 2 abr. 2017.

VIRTUAL WATER. [2017]. Disponível em: <http://virtualwater.eu/>. Acesso em: 25 set. 2017.

WINKLER, Inga T. The human right to water. Great Britain: Hart, 2012.

Patricia Grazziotin Noschang é doutora em Direito pelo Programa de PósGraduação em Direito da Universidade Federal de Santa Catarina (UFSC/2015), Mestre em Direito e Relações Internacionais pelo Programa de Pós-Graduação em Direito da Universidade Federal de Santa Catarina (UFSC/2010). Especialista em Direito Internacional Público, Privado e da Integração Regional pela Universidade Federal do Rio Grande do Sul. MBA em Gestão e Negócios Internacionais pela Fundação Getúlio Vargas. Docente Adjunta da Faculdade de 
Direito da Universidade de Passo Fundo (UPF-2004), Professora do PPGDireito da Universidade de Passo Fundo (UPF).

E-mail: patriciagn@upf.br.

Endereço Postal Institucional: Universidade de Passo Fundo, BR 285, Bairro São José, Passo Fundo, RS. CEP: 99052-900.

Adriana Fasolo Pilati Scheleder é Doutora em Direito pelo Programa de PósGraduação em Direito da Universidade Federal de Santa Catarina (UFSC-2015), Mestre em Direito Processual Civil pela Pontifícia Universidade Católica do Rio Grande do Sul (PUC/RS-2003), Especialista em Direito e Processo Civil pela Universidade do Vale do Rio dos Sinos (UNISINOS-1999), Docente Titular da Faculdade de Direito da Universidade de Passo Fundo (UPF-1999), Professora e Coordenadora Adjunta do PPGDireito da Universidade de Passo Fundo (UPF). E-mail: apilati@upf.br.

Endereço Postal Institucional: Universidade de Passo Fundo, BR 285, Bairro São José, Passo Fundo, RS. CEP: 99052-900. 